Original Research

\title{
Individual Radiosensitivity in Lung Cancer Patients Assessed by G0 and Three Color Fluorescence in Situ Hybridization
}

Theresa Mayo ${ }^{\dagger}$, Barbara Schuster ${ }^{\dagger}$, Anna Ellmann, Manfred Schmidt, Rainer Fietkau, Luitpold V. Distel $^{*}$

Department of Radiation Oncology of the University Hospitals and Friedrich-Alexander-University Erlangen-Nürnberg, Erlangen, Germany; E-Mails: theresa.mayo@web.de;

Barbara.Schuster@extern.uk-erlangen.de; el.an@hotmail.de; Manfred.Schmidt@uk-erlangen.de;

Rainer.Fietkau@uk-erlangen.de; Luitpold.Distel@uk-erlangen.de

$\dagger$ These authors contributed equally to this work.

* Correspondence: Luitpold V. Distel; E-Mail: Luitpold.Distel@uk-erlangen.de

Academic Editor: Thomas Liehr

Special Issue: Applications of Fluorescence in Situ Hybridization

OBM Genetics

2019 , volume 3 , issue 2

doi:10.21926/obm.genet.1902082
Received: July 27, 2018

Accepted: June 13, 2019

Published: June 26, 2019

\begin{abstract}
Background: It is well known that radiosensitivity varies substantially from individual to individual. This may influence the tumor response and also cause side effects in normal tissues following the radiotherapy treatment for cancers. Therefore, we aimed to analyze the sensitivity of a lung cancer cohort to see whether patients display similar radiosensitivity distribution as compared to healthy individuals.

Methods: Blood samples of healthy individuals $(n=244)$ and a small group of lung cancer patients $(n=38)$ were irradiated ex vivo and chromosomes \# 1, 2 and 4 were analyzed by the 3 -color fluorescence in situ hybridization (FISH) technique. Additionally, the data from 400 individuals from previously published studies were also analyzed, which included a healthy cohort as well as rectal cancer patients. Chromosomal aberrations were counted and
\end{abstract}

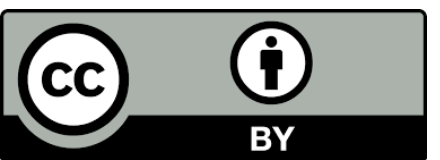

(C) 2019 by the author. This is an open access article distributed under the conditions of the Creative Commons by Attribution License, which permits unrestricted use, distribution, and reproduction in any medium or format, provided the original work is correctly cited. 
expressed as breaks per metaphase $(B / M)$. The chromosomal aberrations in the control sample (background) were subtracted to obtain only the radiation-induced aberrations.

Results: The lung cancer cohort had significantly increased background $B / M$ values $(p<0.001)$. Increased radiosensitivity was stated if the $\mathrm{B} / \mathrm{M}$ value exceeded 0.55 . The percentage of individuals with increased radiosensitivity was slightly higher in the patient groups compared to that in healthy individuals.

Conclusions: Individualizing treatment by adjusting the radiation dose can help in reducing side effects, as cancer patients have a broader range of individual radiosensitivity.

\section{Keywords}

Three color fluorescence in situ hybridization; lung cancer; rectal cancer; individual radiosensitivity; chromosomal aberrations; breaks per metaphase

\section{Introduction}

For many years now it has been known that every individual has a distinct sensitivity to ionizing radiation. These individual differences are especially important when it comes to the use of radiation for cancer treatment.

Some patients with increased radiosensitivity experience extreme side effects during and even years after radiation therapy. In rare cases, these may even cause death [1].

Therefore, testing the individual sensitivity beforehand can offer a major improvement in the therapy. It can help reduce the side effects without decreasing tumor response, by individualizing the treatment dose.

Nevertheless, testing radiation sensitivity poses some difficulties too [2]. While some of the available testing methods cannot really be replicated, yet the biggest problem encountered is transferring of the various tests across different laboratories. There are some standard procedures that have been established for radiosensitivity testing [3]. Dicentric and micronuclei assays are widely accepted tests for the same, though they only take a limited amount of aberrations into account. This, in our opinion, is a clear limitation. Therefore, the 3-color Fluorescence in situ Hybridization (FISH) was preferred, as it represents $23 \%$ of the whole genomic DNA and involves about $34 \%$ of all aberrations. This assay is a tradeoff between using only three easily evaluable chromosomes and simultaneously having a high amount of aberrations [4].

It has been well documented that there are large inter-individual variations in chromosomal aberrations, which can be used as indicators for radiation sensitivity [5-7]. There have been many different approaches like using conventional stain of the chromosomes or the FISH-technique, G0 or $\mathrm{G} 2$ assays and many others. Even though the tests did differ in many ways, the data collected from chromosomal aberrations could predict individual radiosensitivity in a majority of the studies [8-15].

We used a G0-assay in combination with the 3-color-FISH technique to examine the radiationinduced chromosomal aberrations after ex vivo irradiation of lymphocytes in hundreds of individuals including both healthy people and cancer patients. A lung cancer cohort was also studied and the sensitivity was compared with the healthy individuals and an additional rectal 
cancer cohort. We aimed to determine, whether there is any correlation between the radiosensitivity of patients with lung cancer, those with rectal cancer, and healthy individuals.

\section{Materials and Methods}

\subsection{Patients}

Lymphocytes from 38 patients who had received radiotherapy in some parts of the lungs were examined and compared to a cohort of 244 healthy individuals divided into two subgroups, one was gender and age-matched, while the other was not (Table 1). In addition, the results of this study were compared to the previously acquired data on a cohort of 220 rectal cancer patients and 180 healthy individuals [3, 5]. Increased radiosensitivity was acknowledged if the value of the chromosomal aberrations exceeded $0.55 \mathrm{~B} / \mathrm{M}$. Blood samples (heparinized) were drawn prior to the beginning of the treatment.

Table 1 The demographic and clinical characteristics of patients (percentage).

\begin{tabular}{|c|c|c|c|}
\hline & Patients & Age matched & Non-matched \\
\hline & & \multicolumn{2}{|c|}{ healthy individuals } \\
\hline $\mathbf{n}$ & 38 & 31 & 213 \\
\hline \multicolumn{4}{|l|}{ Gender (\%) } \\
\hline Male & $29(76)$ & $22(71)$ & $94(44)$ \\
\hline Female & $9(24)$ & $9(29)$ & $119(56)$ \\
\hline \multicolumn{4}{|l|}{ Age (years) } \\
\hline Mean age & 65.2 & 65.8 & 50.7 \\
\hline Range & $49-83$ & $50-81$ & $9-81$ \\
\hline \multicolumn{4}{|l|}{ Type of therapy (\%) } \\
\hline only RT & $3(8)$ & & \\
\hline sim. RCT & $35(92)$ & & \\
\hline CT before RT/RCT & $6(13)$ & & \\
\hline Pre-RT (\%) & $16(35)$ & & \\
\hline
\end{tabular}

\subsection{Chromosomal Preparation}

The blood sample of each individual was divided into two parts; one was irradiated with a dose of $2 \mathrm{~Gy}$ by a 6-MV linear accelerator (Mevatron, Siemens, Germany), while the other was left as a control. Lymphocytes were then stimulated with phytohemagglutinin and incubated for $48 \mathrm{~h}$. Mitosis was blocked by the addition of colcemid and chromosome preparation was performed, in accordance with the standard protocol. Subsequently, chromosomes \# 1, 2 and 4 were stained with different fluorescent dyes and the whole DNA was counterstained with DAPI. The fluorescence in situ hybridization (FISH) was carried out as described previously [7, 16, 17]. 


\subsection{Image Acquisition and Analysis}

The chromosomal aberrations were scored using a fluorescence microscope (Zeiss, Axioplan 2, Goettingen, Germany) and the Metasystem software (Metafer 4 V3.10.1, Altlussheim, Germany), both of which were used to search for and acquire an image of every metaphase. These images were then analyzed with the help of an image analyzing software (Biomas, Erlangen, Germany) and the different aberrations evident within the stained chromosomes were scored on the basis of the number of underlying chromosomal breakages according to Savage and Simpson [17]. For a better comparison, these were expressed as breaks per metaphase (B/M) [18]. The B/M value of the irradiated blood sample was then corrected by the way of subtraction of the breaks per metaphase in the control sample (background).

\subsection{Statistical Analysis}

Statistical analysis of the data was performed using the SPSS Statistics 21 software (IBM, Armonk, NY, USA). Statistical significance was tested using t- and Levene's tests. The graphs were plotted in MS-Excel (Microsoft Corporation, Redmond, WA, USA).

This study was approved by the ethics review committee of the Friedrich-AlexanderUniversitaet Erlangen-Nuernberg (No. 2725). Written informed consent was obtained from all the patients.

\section{Results}

The irradiation-induced aberrations in the stained chromosomes \#1, 2 and 4 were thoroughly studied by the authors, as these chromosomes alone make up $22 \%$ of the entire genome and thus have high explanatory power (Figure 1a). The use of the three color fluorescence in situ hybridization (FISH) of these chromosomes provides the opportunity to distinguish not only aberrations like di- or acentric chromosomes but also translocations or insertions, which would have otherwise been missed [19-25] (Figure 1b). The number of breaks per aberration was summed up, as described by Savage et al., and divided by the number of all scored metaphases $(B / M)[17,18]$. The analyzed number of metaphases per individual varied quite widely, ranging from 47 to 504 metaphases analyzed per individual. Overall, the average number of metaphases scored and analyzed came out to be 183 for each person.

The reason for using the $\mathrm{GO}$ assay is that all lymphocytes are located in the $\mathrm{GO}$ phase of the cell cycle. Thus all the cells examined were independent of the cell cycle phase-dependent radiosensitivity, which varies by a factor of 2.5 between late $S$ phase and $G 2$ phase (Figure 1c). After irradiation, cell division was stimulated by phytohemagglutinin. This means that the cells repair the induced DNA damage mainly in the $\mathrm{GO}$ phase and to a lower extent, after entering the cell cycle. The cells have to pass all the checkpoints during this assay and if DNA damage is not sufficiently repaired, they can either be arrested or inactivated. If not so, the cells are arrested by colcemid at the first entry into mitosis phase, after the irradiation and stimulation.

At first, a cohort of healthy individuals, called healthy individuals $I$, and rectal cancer patients was analyzed for better comparison in order to identify a cut-off value for increased individual radiosensitivity. The non-irradiated samples were used only to subtract the background from the ex vivo irradiated samples. Nevertheless, it is obvious that cancer patients have more 
chromosomal aberration background rates compared to healthy individuals (Figure 2a). In the ex vivo irradiated sample, the Gaussian distributions of healthy individuals were very similar to those of the rectal cancer patients (Figure 2b). To specify which patients are rated as having an increased radiosensitivity, a threshold for increased radiosensitivity is required. Using the 1.5-time standard deviation, a B/M value of 0.55 can be used as an adequate cut-off for increased radiosensitivity in individuals (Table 2). Considering this fact, the study included a total of 30 (13.6\%) rectal cancer patients with increased radiosensitivity; whereas, within the healthy cohort I, only 14 (7.8\%) individuals displayed $\mathrm{B} / \mathrm{M}$ values above 0.55 .
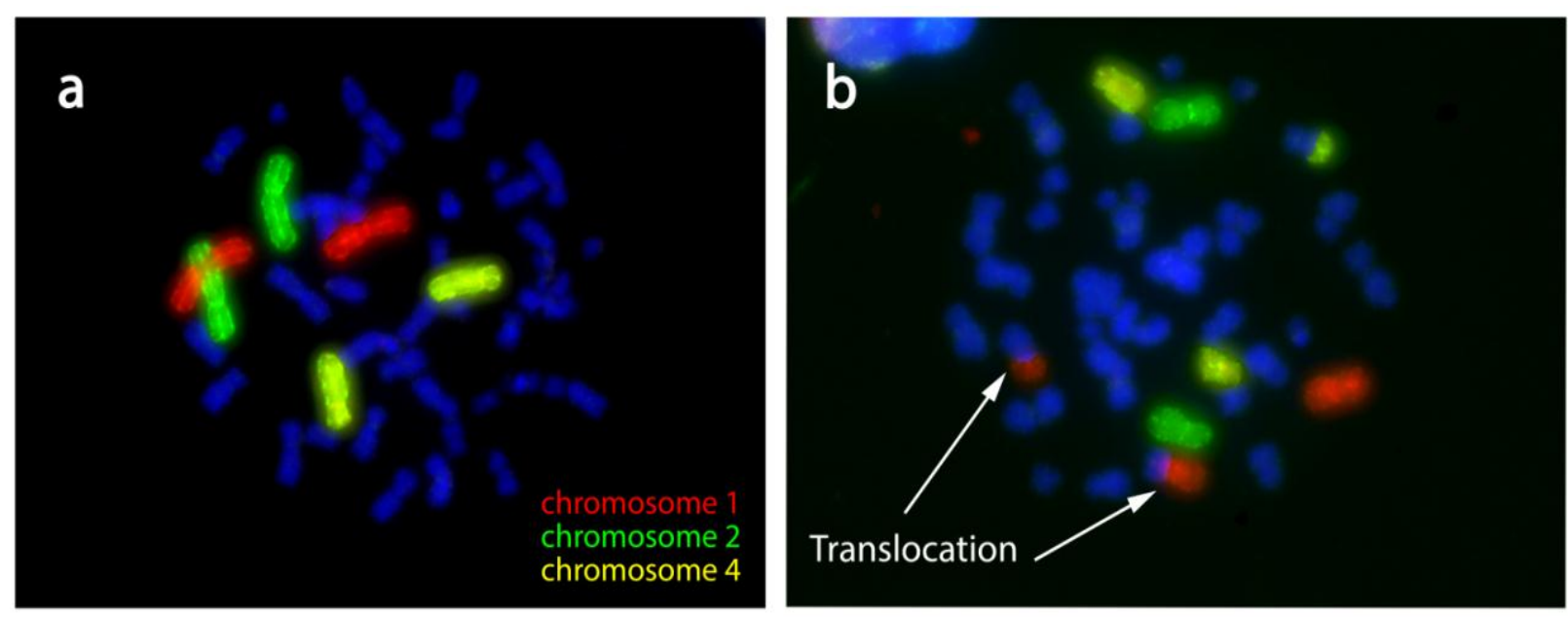

C
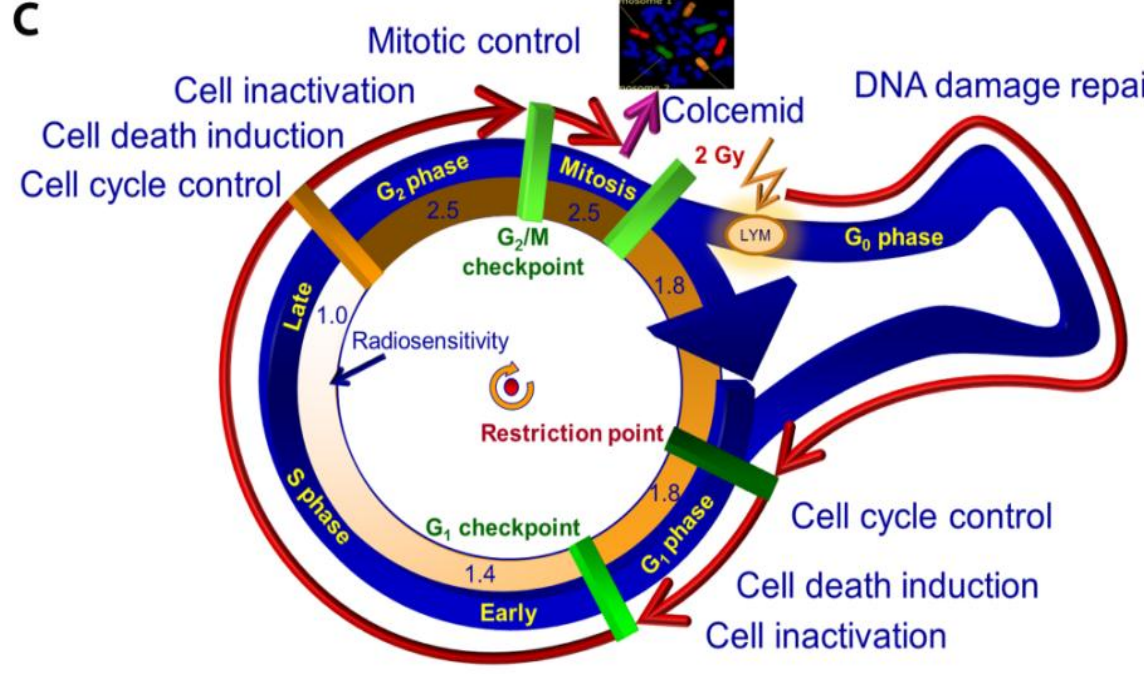

Figure 1 Three-color fluorescence in situ hybridization. Metaphase spreads of human blood lymphocytes with chromosomes \# 1 (red), \# 2 (green) and \# 4 (yellow) stained with a chromosome-specific probe. DNA was counterstained with DAPI (blue) (a+b). Normal metaphase spread (a). Metaphase spread with two translocations of chromosome \#1 and \#2 with a blue chromosome (b). This was scored as four breaks. Cell cycle illustration with checkpoints and radiation sensitivity during the different cell cycle phases (c). The time point when the blood lymphocytes get irradiated (orange arrow) and the phases and checkpoints that the cells have to pass through during the cell cycle (red arrow). Cell cycle arrested using colcemid to perform the 3-C FISH (purple arrow). 
a

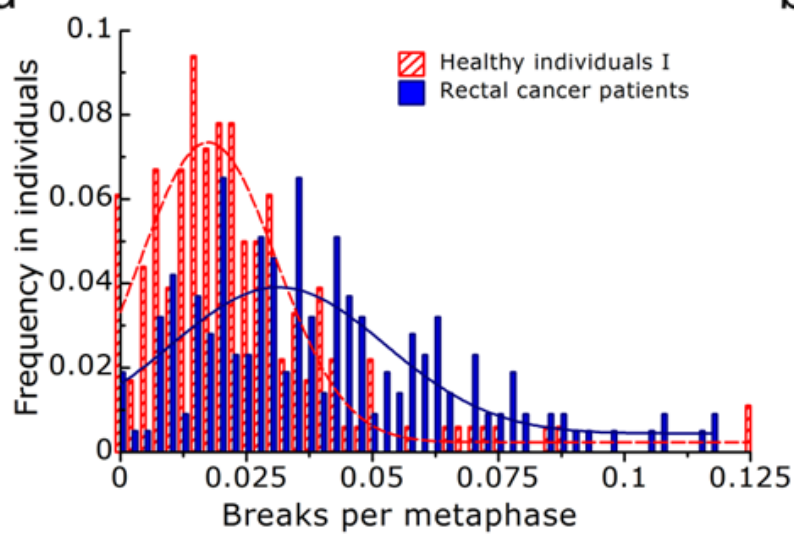

$\mathrm{C}$

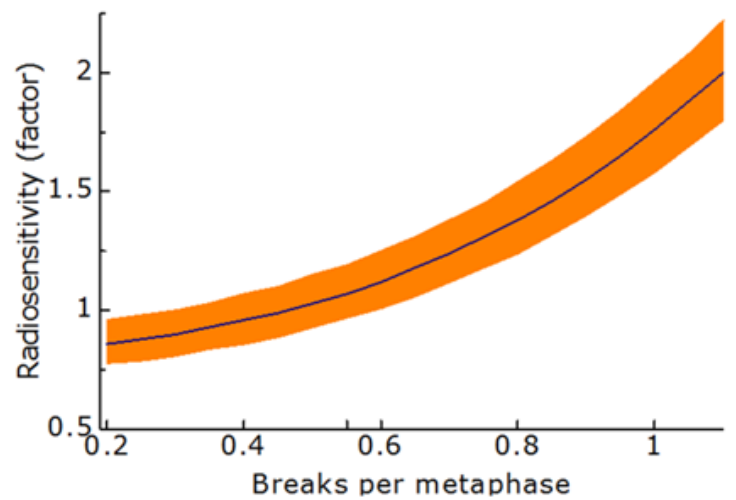

Breaks per metaphase: $\begin{array}{lllllllll}0.2 & 0.3 & 0.4 & 0.5 & 0.6 & 0.7 & 0.8 & 0.9 & 1\end{array}$

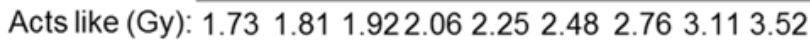

Dose adaption (Gy): $2.32 \quad 2.212 .091 .941 .781 .611 .451 .291 .14$

Figure 2 Comparison of the frequency of individuals having a distinct $B / M$ value from a group of healthy individuals with a group of rectal cancer patients. B/M in the untreated background blood sample (a) and after ex vivo irradiation with 2 Gy. Background values are subtracted (b). A cut-off value of $0.55 \mathrm{~B} / \mathrm{M}$ is marked with a purple bar (b). Matching of distinct $B / M$ values with the corresponding radiosensitivity. The comparison of the $\mathrm{B} / \mathrm{M}$ values and the dose effect of irradiating with $2 \mathrm{~Gy}$ on the individual, along with the necessary dose adjustment to achieve the desired effect of 2 Gy (c).

Table 2 Mean value and standard deviation of the Gaussian distributions.

\begin{tabular}{rccccccc}
\hline & & & 2 Gy & & \\
& Bntreated & & Mean + & & & Mean + \\
& SD & $\mathbf{1 . 5}$ x SD & B/M & SD & $\mathbf{1 . 5}$ x SD \\
\hline Healthy individuals I & 0.017 & 0.014 & 0.038 & 0.39 & 0.09 & 0.54 \\
Cancer patients & 0.031 & 0.021 & 0.063 & 0.40 & 0.10 & 0.56 \\
Healthy individuals II & 0.034 & 0.009 & 0.048 & 0.407 & 0.095 & 0.55 \\
Matched healthy & 0.042 & 0.016 & 0.067 & 0.449 & 0.088 & 0.58 \\
individuals & & & & & & \\
Lung cancer patients & 0.090 & 0.039 & 0.149 & 0.382 & 0.139 & 0.59 \\
\hline
\end{tabular}


The patients having an increased radiosensitivity attain a higher amount of non-processed or misprocessed damage to their cells as compared to the patients with an average radiosensitivity. Thus there is an increased risk of unwanted effects from therapeutic treatment. In order to overcome this problem, empirical values were used to create a calibration curve for radiosensitivity (Figure 2c). This was established by using known radiosensitivities from different cohorts. In one case, the data from patients with heritable radiosensitivity syndromes such as 'ataxia telangiectasia' and 'Nijmegen breakage syndrome' were used, which at one B/M has an assumed radiosensitivity of 2 . This means that any irradiation would have double the effect on such patients than it would have on an average healthy individual. In addition, the data from healthy individuals and cancer patients were also taken into consideration to have a score for an average radiosensitivity. This equivalent was considered as the mean value $(0.4 \mathrm{~B} / \mathrm{M})$. A threshold for the adjustment of radiotherapy dose at the mean $B / M$ of +1.5 times standard deviation, resulting in $0.55 \mathrm{~B} / \mathrm{M}$ was adopted. It was assumed that below this threshold, the sensitivity is rather moderate in variation and $\mathrm{B} / \mathrm{M}$ rises only slightly. Consequently, the slope has to be steeper between the $0.55 \mathrm{~B} / \mathrm{M}$ threshold and the 2 times increased radiosensitivity at $1 \mathrm{~B} / \mathrm{M}$. This radiosensitivity factor, derived from the curve, multiplied by the treatment dose, provides the actual dose taking effect on the cells of a radiosensitive individual as compared to that of an average sensitive individual. In order to determine the needed dose, the reciprocal value of the factor multiplied by the desired dose is taken as the adapted dose (Figure $2 \mathrm{c}$ ).

This adequate dose adaptation is important, especially in radiation therapy of cancer. All the lung cancer patients participating in this study received this treatment as we wanted to know if this cohort displays a higher rate of radiosensitive individuals who would need a dose adaptation. In comparison to the second analyzed group of the healthy individuals, cohort II, who was divided into age-matched and a non-matched healthy control groups, the first noticeable difference was the much higher rate of background chromosomal aberration $(p<0.001)$ (Figure 3a). This was similar to the rectal cancer cohort mentioned earlier, though at a much higher level. Both the cohorts of healthy individuals showed fewer metaphase breaks while the non-matched cohort presented the lowest number of metaphase breaks. In the ex vivo irradiated sample, the distribution within the cancer patients' cohort was broader in comparison to each of the healthy individuals' cohorts (Figure $3 \mathrm{~b}$ ). The age-matched control group displayed slightly higher values than the non-matched group, while their distributions were still narrower than the cancer patients' cohort. The percentage of individuals with increased radiosensitivity among the cancer patients was $16 \%$, with 6 out of 38 individuals having $B / M$ above 0.55 . In the age-matched healthy cohort, there were $13 \%$ radiosensitive individuals while in the unmatched cohort, $10 \%$ (22 out of 213) individuals were radiosensitive.

The lung cancer cohort had a significantly higher mean $\mathrm{B} / \mathrm{M}$ value and a much broader range in the un-irradiated control sample (matched: $0.035 \mathrm{~B} / \mathrm{M}$; non-matched: $0.025 \mathrm{~B} / \mathrm{M}$; BC: $0.109 \mathrm{~B} / \mathrm{M}$; $p<0.001$ ) (Figure $3 c$ ). The healthy individuals, on the other hand, had a slightly higher though not significant $\mathrm{B} / \mathrm{M}$ value after irradiation (matched: $0.457 \mathrm{~B} / \mathrm{M}$; non-matched: $0.422 \mathrm{~B} / \mathrm{M}$; $\mathrm{BC}$ : 0.403 $\mathrm{B} / \mathrm{M}$ ) (Figure 3b). 
a

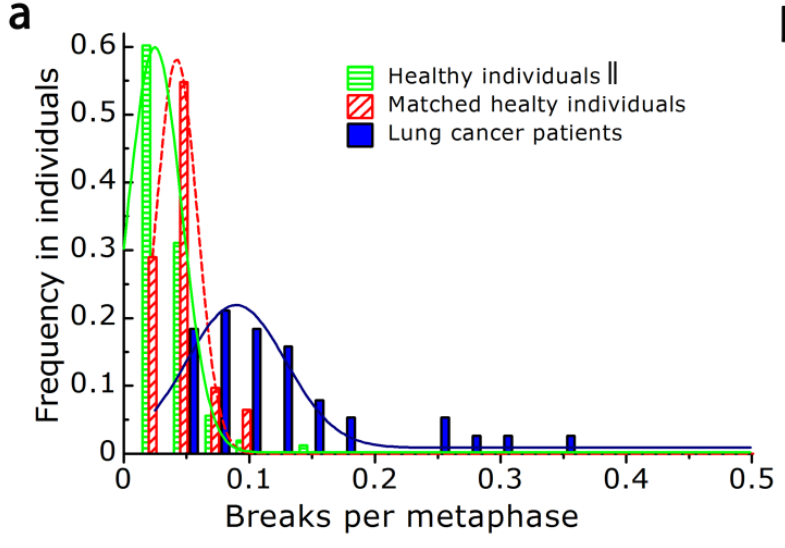

C

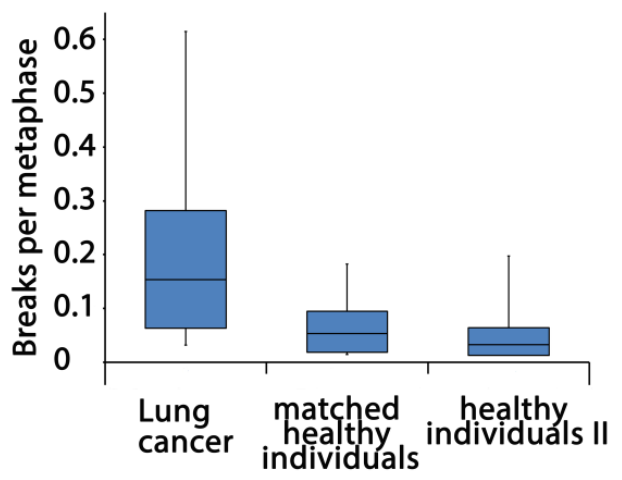

b

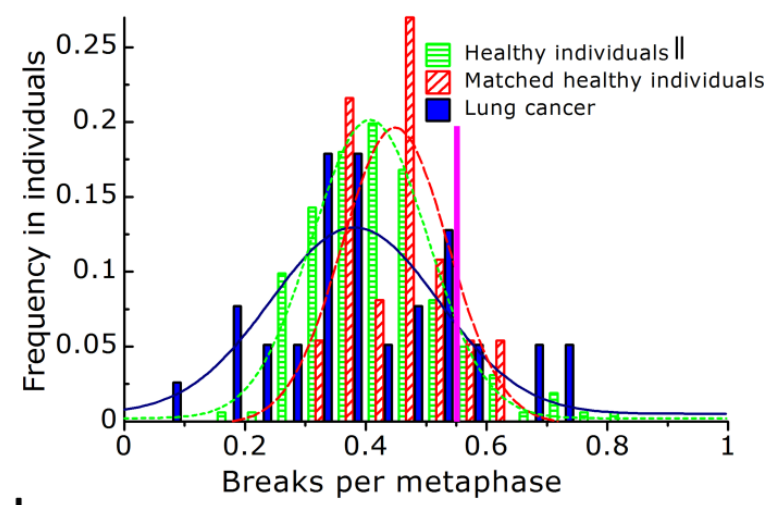

d

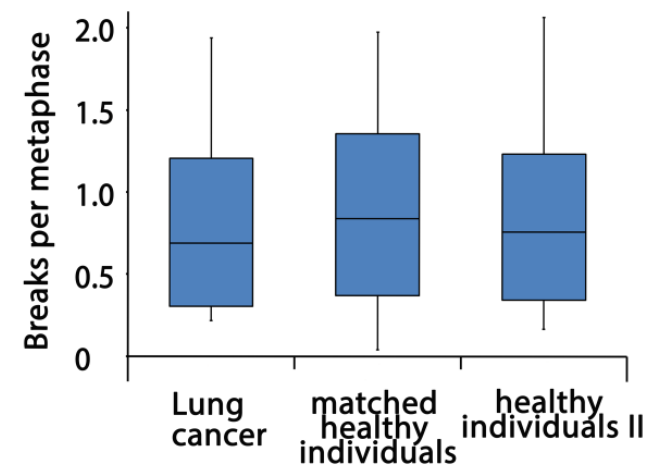

Figure 3 Comparison of the $\mathrm{B} / \mathrm{M}$ values in the cohort of lung cancer patients with healthy age-matched and non-matched individuals in non-irradiated lymphocytes (a) and in lymphocytes ex vivo irradiated with $2 \mathrm{~Gy}(\mathrm{~b})$. The cut-off value of $0.55 \mathrm{~B} / \mathrm{M}$ is marked in purple. The spontaneous breaks per metaphase were subtracted from the latter. Boxplot of the scored B/M values in the same groups $(c+d)$. Significant differences in the control blood sample, no significant differences after irradiation.

\section{Discussion}

Since there is a high inter-individual difference in sensitivity to ionizing radiation, it is important to find a reliable and standardized test for its evaluation, especially before implementing any kind of treatment, including radiation therapy. Different methods have been employed for this purpose and several studies have used lymphocytes as a predictor $[2,8,12,13,15,26]$. It has been previously stated that the difficulty with testing for sensitivity is that no standardized protocol has been established as yet [2].

One method that has been demonstrated for testing individual radiosensitivity is the 3-colorFISH, which has been proven to be very reliable $[6,16]$. Its advantage is that the lymphocytes can be appropriated from peripherally drawn blood. In addition, the cells are irradiated in the G0 phase, which can anticipate any difference in the cell cycle-dependent radiosensitivity. Even though chromosomal aberrations are induced by radiation, an equal amount of cells go into mitosis as compared to the non-irradiated ones [7]. After receiving a dose of ionizing radiation, the lymphocytes have to repair the induced DNA damage and pass through the entire cell cycle to get into mitosis phase (Figure $2 \mathrm{c}$ ). Therefore, any impairment in the DNA repair proteins may cause residual chromosomal aberrations which can be detected via the 3-C-FISH. Consequently, 
individual $\mathrm{B} / \mathrm{M}$ value can be used as a good predictor of radiation sensitivity. The advantage of using fluorescent dyes for chromosome \#1, 2 and 4 is that, in addition to the acentric and dicentric chromosomes, translocations and minor insertions of about $5 \mathrm{Mbp}$ can also be identified [23]. The three chromosomes account to $22 \%$ of the whole genome and can carry up to $34 \%$ of all aberrations [21, 25]. Further, the most suitable dose for identifying any sensitive patient is supposed to be $2 \mathrm{~Gy}$. 'Breaks per metaphase' is the parameter having the highest statistical power for its detection [7]. Staining of all 24 chromosomes would be too challenging and analysis of the aberrations would be far too complex, considering the purpose as only to develop a simple tool for evaluation of radiosensitivity [25]. This test has been validated by examining hundreds of individual samples over many years $[6,16]$. In comparison, other tests attempting to measure the individual response to ionizing radiation do not seem to be able to be replicated as yet [27]. The sole disadvantage of this approach is that the procedure is time-consuming, apart from the fact that the evaluation of the metaphases requires some experience.

In order to measure $\mathrm{B} / \mathrm{M}$ values in a radiotherapeutic treatment, it is important to know the correct interpretation of specific data. Therefore, a dose adjustment curve was introduced in this study, by analyzing patients with heritable increased radiosensitivity as well as healthy individuals (Figure 2c). An important fact to be understood is that the adjustment cannot be done in a linear manner, but has to be either decreased or increased gradually (Figure 2c). Since it is based on empirical values there is a slight uncertainty but within an acceptable range. This enables the possibility of adjustment of the applied dose in radiotherapy according to individual response. In this way, the tumor response is maintained while the side effects are minimized. By using this dose adjustment and individualizing therapy, a decrease in the incidents of acute and chronic side effects arising from ionizing radiations can be established. Also, the non-cancer effects of this treatment such as cardiovascular diseases and secondary malignancies may be prevented [28].

As a higher incidence of chromosomal aberrations was found in cancer patients of younger age as compared to a healthy cohort, the latter particularly is very important in younger individuals [3]. An impairment in certain repair proteins may cause genomic instability due to incorrectly repaired DNA [27], which may lead to accumulation of mutations and chromosomal aberrations in particular, which in turn may increase the occurrence of malignancies [8, 29]. Up to $20 \%$ of all cancers are thought to be having a genetic background, either caused by high penetrance or low penetrance genes with polygenetic mechanisms [30]. Thus, there exists a link between chromosomal aberrations and the development of cancer. It was postulated that up to $35 \%$ of the genes responsible for radiation sensitivity are also accountable for tumorigenesis $[3,5]$. This explains why the individual inability to prevent chromosomal aberrations is an important disposition for the development of malignant cells $[5,27]$. Furthermore, this may be an explanation for the increased rate of spontaneous $\mathrm{B} / \mathrm{M}$ in the lung cancer cohort in comparison to the healthy individuals, observed in this study. Other studies have also investigated an increased rate of accumulated chromosomal aberrations in their cohorts of prostate and breast cancer patients $[29,31]$.

Since the processing of impaired DNA damage makes individuals more susceptible to cancer, a higher number of sensitive patients above the cut-off of $0.55 \mathrm{~B} / \mathrm{M}$ was expected [32]. Certainly, a slightly higher percentage of lung cancer patients with increased radiosensitivity was detected in comparison to healthy individuals. However, these differences were lower than expected, as other cancer cohorts like breast cancer patients demonstrated more individuals above the cut-off value 
[29]. Yet, it cannot be said that only genes are important in determining individual radiosensitivity. It is well-known that radiation response is also influenced by other factors such as age, smoking, and diabetes [33]. These environmental and lifestyle factors also have the ability to induce chromosomal aberrations and may lead to tumor induction [5].

Keeping this in mind, the clearly increased mean $B / M$ value within the lung cancer patients in non-irradiated blood samples could be a clue for the influence of lifestyle factors such as tobacco use. Smoking tobacco is closely associated with the development of lung cancer and has an increased mutation rate, not only in smoking-related cancers but even in the non-cancerous lung tissues of smokers $[34,35]$. This supports the idea that lung cancer patients are less radiosensitive than patients with other types of cancers. The reason may be that, even in individuals with a genetically good disposition, mutations and, as a consequence, cancer is caused by the presence of high amounts of noxious substances.

The slightly lower $\mathrm{B} / \mathrm{M}$ values after irradiation in the patients in this study could be explained by the aforementioned fact as the background chromosomal aberrations prior to irradiation were subtracted. Nevertheless, it may also be possible that the ionizing radiation induces several additional aberrations so that the lymphocytes are not able to pass the cell cycle checkpoints (Figure 1c) and eventually go into apoptosis. Therefore, fewer cells with high aberration counts would be included.

However, it is not that only genetic or environmental factors have an impact on the number of chromosomal aberrations. The age-matched group of healthy individuals had a slightly increased $\mathrm{B} / \mathrm{M}$ rate as compared to the overall control group. The reason might be that on anerage, the matched controls were older (66 vs. 51 years) than the general healthy cohort and the fact that the radiosensitivity seems to increase with age in cohorts of healthy individuals [3].

\section{Conclusions}

Among cancer patients, a certain subgroup is distinctly more sensitive to ionizing radiation than the average radiosensitive patients. Thus, individualized treatment by testing sensitivity beforehand could help achieve a better outcome in every patient. Using a standardized test like the 3-C-FISH could be a reliable way of implementing this process. Furthermore, the influence of noxious substances rather than increased radiosensitivity, especially within lung cancer cohort, may be the determining factor in tumorigenesis. Since only a small sample size was examined in this study, further evaluation of these findings with more patients is warranted.

\section{Acknowledgments}

The author would like to thank Doris Mehler and Elisabeth Müller for excellent technical support for the study and all individuals for the donations of blood.

\section{Author Contributions}

TM generated the data, part of the statistical analyses and drafted the manuscript. LD performed the statistical analysis, illustrated most figures and finalized the manuscript. Part of the data was generated by BS and AE (rectal carcinoma patients). RF and MS designed and enabled this study. All authors read and approved the final manuscript. 


\section{Competing Interests}

The authors have declared that no competing interests exist.

\section{References}

1. Fahrig $A$, Koch $T$, Lenhart $M$, Rieckmann $P$, Fietkau $R$, Distel $L$, et al. Lethal outcome after pelvic salvage radiotherapy in a patient with prostate cancer due to increased radiosensitivity: Case report and literature review. Strahlenther Onkol. 2018; 194: 60-66.

2. Rajaraman $P$, Hauptmann $M$, Bouffler $S$, Wojcik A. Human individual radiation sensitivity and prospects for prediction. Ann ICRP. 2018; 47: 126-141.

3. Schuster B, Ellmann A, Mayo T, Auer J, Haas M, Hecht M, et al. Rate of individuals with clearly increased radiosensitivity rise with age both in healthy individuals and in cancer patients. BMC Geriatr. 2018; 18: 105.

4. Kuechler A, Neubauer S, Grabenbauer GG, Claussen U, Liehr T, Sauer R, et al. Is 24-color fish detection of in-vitro radiation-induced chromosomal aberrations suited to determine individual intrinsic radiosensitivity?. Strahlenther Onkol. 2002; 178: 209-215.

5. Distel LV, Neubauer S, Keller U, Sprung CN, Sauer R, Grabenbauer GG. Individual differences in chromosomal aberrations after in vitro irradiation of cells from healthy individuals, cancer and cancer susceptibility syndrome patients. Radiother Oncol. 2006; 81: 257-263.

6. Gebhart E, Neubauer S, Schmitt G, Birkenhake S, Dunst J. Use of a three-color chromosome in situ suppression technique for the detection of past radiation exposure. Radiat Res. 1996; 145: 47-52.

7. Keller U, Kuechler A, Liehr T, Muller E, Grabenbauer G, Sauer R, et al. Impact of various parameters in detecting chromosomal aberrations by fish to describe radiosensitivity. Strahlenther Onkol. 2004; 180: 289-296.

8. Baeyens A, Thierens H, Claes K, Poppe B, Messiaen L, De Ridder L, et al. Chromosomal radiosensitivity in breast cancer patients with a known or putative genetic predisposition. $\mathrm{Br} J$ Cancer. 2002; 87: 1379-1385.

9. Barber JB, Burrill W, Spreadborough AR, Levine E, Warren C, Kiltie AE, et al. Relationship between in vitro chromosomal radiosensitivity of peripheral blood lymphocytes and the expression of normal tissue damage following radiotherapy for breast cancer. Radiother Oncol. 2000; 55: 179-186.

10. Baria K, Warren C, Roberts SA, West CM, Scott D. Chromosomal radiosensitivity as a marker of predisposition to common cancers?. Br J Cancer. 2001; 84: 892-896.

11. Beaton LA, Marro L, Samiee S, Malone S, Grimes S, Malone K, et al. Investigating chromosome damage using fluorescent in situ hybridization to identify biomarkers of radiosensitivity in prostate cancer patients. Int J Radiat Biol. 2013; 89: 1087-1093.

12. Hoeller $U$, Borgmann $K$, Bonacker $M$, Kuhlmey A, Bajrovic A, Jung $H$, et al. Individual radiosensitivity measured with lymphocytes may be used to predict the risk of fibrosis after radiotherapy for breast cancer. Radiother Oncol. 2003; 69: 137-144.

13. Huber R, Braselmann H, Geinitz H, Jaehnert I, Baumgartner A, Thamm R, et al. Chromosomal radiosensitivity and acute radiation side effects after radiotherapy in tumour patients--a follow-up study. Radiat Oncol. 2011; 6: 32. 
14. Popanda O, Ebbeler R, Twardella D, Helmbold I, Gotzes F, Schmezer P, et al. Radiationinduced DNA damage and repair in lymphocytes from breast cancer patients and their correlation with acute skin reactions to radiotherapy. Int J Radiat Oncol Biol Phys. 2003; 55: 1216-1225.

15. Scott D, Spreadborough AR, Jones LA, Roberts SA, Moore CJ. Chromosomal radiosensitivity in g2-phase lymphocytes as an indicator of cancer predisposition. Radiat Res. 1996; 145: 3-16.

16. Neubauer S, Dunst J, Gebhart E. The impact of complex chromosomal rearrangements on the detection of radiosensitivity in cancer patients. Radiother Oncol. 1997; 43: 189-195.

17. Savage JR, Simpson P. On the scoring of fish-"painted" chromosome-type exchange aberrations. Mutat Res. 1994; 307: 345-353.

18. Keller U, Grabenbauer G, Kuechler A, Sauer R, Distel L. Technical report. Radiation sensitivity testing by fluorescence in-situ hybridization: How many metaphases have to be analysed? Int J Radiat Biol. 2004; 80: 615-620.

19. Dunst J, Neubauer S, Becker A, Gebhart E. Chromosomal in-vitro radiosensitivity of lymphocytes in radiotherapy patients and at-homozygotes. Strahlenther Onkol. 1998; 174: 510-516.

20. Johannes $\mathrm{C}$, Chudoba I, Obe $\mathrm{G}$. Analysis of $\mathrm{x}$-ray-induced aberrations in human chromosome 5 using high-resolution multicolour banding fish (mband). Chromosome Res. 1999; 7: 625-633.

21. Kuechler A, Dreidax M, Pigorsch SU, Liehr T, Claussen U, Wendt TG, et al. Residual chromosomal damage after radiochemotherapy with and without amifostine detected by 24 color fish. Strahlenther Onkol. 2003; 179: 493-498.

22. Neubauer S, Gebhart E, Schmitt G, Birkenhake S, Dunst J. Is chromosome in situ suppression (ciss) hybridization suited as a predictive test for intrinsic radiosensitivity in cancer patients?. Int J Oncol. 1996; 8: 707-712.

23. Schilling S, Keller U, Sprung CN, Weise A, Grabenbauer GG, Sauer R, et al. Breakpoint locations within chromosomes 1,2 , and 4 of patients with increased radiosensitivity. Cancer Genet Cytogenet. 2006; 168: 1-10.

24. Stumm M, Neubauer S, Keindorff S, Wegner RD, Wieacker P, Sauer R. High frequency of spontaneous translocations revealed by fish in cells from patients with the cancer-prone syndromes ataxia telangiectasia and nijmegen breakage syndrome. Cytogenet Cell Genet. 2001; 92: 186-191.

25. Tucker JD, Ramsey MJ, Lee DA, Minkler JL. Validation of chromosome painting as a biodosimeter in human peripheral lymphocytes following acute exposure to ionizing radiationin vitro. Int J Radiat Biol. 2009; 64: 27-37.

26. Ruckert M, Deloch L, Fietkau R, Frey B, Hecht M, Gaipl US. Immune modulatory effects of radiotherapy as basis for well-reasoned radioimmunotherapies. Strahlenther Onkol. 2018; 194: 509-519.

27. Cavusoglu K, Arica SC, Bokesoy I, Kurtman C. Chromosomal aberrations induced by radiotherapy in lymphocytes from patients with lung cancer. J Environ Biol. 2009; 30: 7-10.

28. Jung H, Beck-Bornholdt HP, Svoboda V, Alberti W, Herrmann T. Quantification of late complications after radiation therapy. Radiother Oncol. 2001; 61: 233-246.

29. Kulzer L, Rubner Y, Deloch L, Allgauer A, Frey B, Fietkau R, et al. Norm- and hypo-fractionated radiotherapy is capable of activating human dendritic cells. J Immunotoxicol. 2014; 11: 328336. 
30. Nagy R, Sweet K, Eng C. Highly penetrant hereditary cancer syndromes. Oncogene. 2004; 23: 6445-6470.

31. Auer J, Keller U, Schmidt M, Ott O, Fietkau R, Distel LV. Individual radiosensitivity in a breast cancer collective is changed with the patients' age. Radiol Oncol. 2014; 48: 80-86.

32. Moses RE. DNA damage processing defects and disease. Annu Rev Genomics Hum Genet. 2001; 2: 41-68.

33. Morgan WF. Human radiosensitivity. Int J Radiat Biol. 2013; 89: 1002-1002.

34. Gibbons DL, Byers LA, Kurie JM. Smoking, p53 mutation, and lung cancer. Mol Cancer Res. 2014; 12: 3-13.

35. Hussain SP, Amstad P, Raja K, Sawyer M, Hofseth L, Shields PG, et al. Mutability of p53 hotspot codons to benzo(a)pyrene diol epoxide (bpde) and the frequency of p53 mutations in nontumorous human lung. Cancer Res. 2001; 61: 6350-6355.

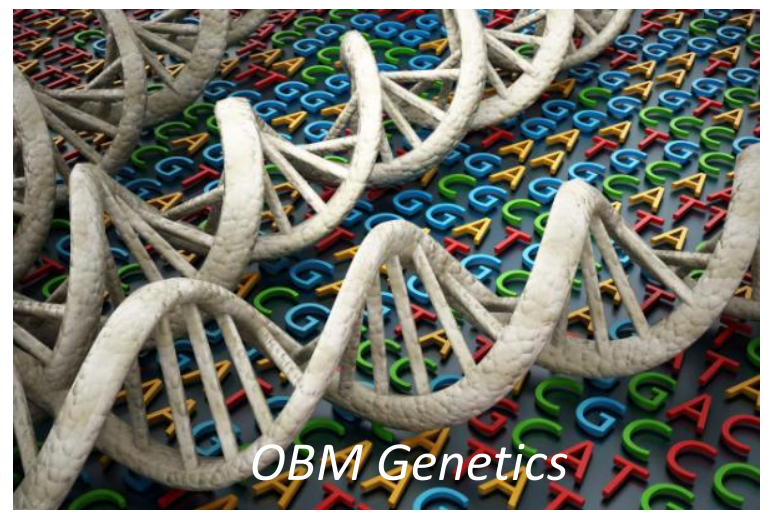

Enjoy OBM Genetics by:

1. Submitting a manuscript

2. Joining in volunteer reviewer bank

3. Joining Editorial Board

4. Guest editing a special issue

For more details, please visit:

http://www.lidsen.com/journals/genetics 